\title{
Lucky Imaging of M subdwarfs ^
}

\author{
N. Lodieu ${ }^{1}$, M. R. Zapatero Osorio ${ }^{1}$, and E. L. Martín ${ }^{1}$ \\ Instituto de Astrofísica de Canarias, Calle Vía Láctea s/n, 38205 La Laguna, Tenerife, Spain \\ e-mail: [nlodieu; mosorio; ege]@iac.es
}

Received 23 January 2009 / Accepted 2 March 2009

\begin{abstract}
Context. The knowledge of the binary properties of metal-poor and solar-metallicity stars can shed light on the potential differences between the formation processes responsible for both types of objects.

Aims. The aim of the project is to determine the binary properties (separation, mass ratio, frequency of companions) for M subdwarfs, the low-metallicity counterparts of field $\mathrm{M}$ dwarfs, and investigate any potential differences between both populations.

Methods. We have obtained high-resolution imaging in the optical for a sample of 24 early-M subdwarfs and nine extreme subdwarfs with the "Lucky Imaging" technique using the AstraLux instrument on the Calar Alto 2.2-m telescope.

Results. We are sensitive to companions at separations larger than $0.1 \operatorname{arcsec}$ and differences of $\sim 2$ mag at 0.1 arcsec and $\sim 5$ mag at 1 arcsec. We have found no companion around the 24 subdwarfs under study and one close binary out of nine extreme subdwarfs. A second image of LHS 182 taken three months later with the same instrument confirms the common proper motion of the binary separated by about 0.7 arcsec. Moreover, we do not confirm the common proper motion of the faint source reported by Riaz and collaborators at $\sim 2$ arcsec from LHS 1074.

Conclusions. We derive a binary frequency of $3 \pm 3 \%$ for $\mathrm{M}$ subdwarfs from our sample of 33 objects for separations larger than about five astronomical units. Adding to our sample the additional 28 metal-poor early-M dwarfs observed with the Hubble Space Telescope by Riaz and collaborators, we infer a binary fraction of $3.7 \pm 2.6 \%$ (with a $1 \sigma$ confidence limit), significantly lower than the fraction of resolved binary M dwarfs ( 20\%) over the same mass and separation ranges. This result suggests a sharp cut-off in the multiplicity fraction from $\mathrm{G}$ to $\mathrm{M}$ subdwarfs, indicating that the metallicity plays a role at lower masses and/or an environmental effect governing the formation of metal-poor $\mathrm{M}$ dwarfs compared to their metallicity counterparts.
\end{abstract}

Key words. methods: observational - techniques: photometric - Galaxy: halo - stars: binaries: general stars: low-mass, brown dwarfs - infrared: stars

\section{Introduction}

Cool subdwarfs are metal-poor dwarfs which appear less luminous than their solar-metallicity counterparts because their atmospheres are deficient in metals (Baraffe et al. 1997). They exhibit halo kinematics, high proper motions and high heliocentric velocities (Gizis 1997). They are typically very old (10 Gyr or more) and likely belong to the first generations of stars in the Galaxy. The adopted classification for M subdwarfs (sdM) and extreme subdwarfs (esdM) originally proposed by Gizis (1997) has recently been revised by Lépine et al. (2007a). A new class, the ultra-subdwarfs (usdM), has been added to the sdM and esdM originally proposed. The new scheme is based on a parameter, $\zeta_{\mathrm{TiO} / \mathrm{CaH}}$, a proxy for the weakening of the strength of the $\mathrm{TiO}$ band as a function of metallicity. An alternative classification scheme has been proposed by Jao et al. (2008) by comparing model grids with optical spectra to characterise the spectral energy distribution of subdwarfs by three parameters: temperature, gravity, and metallicity.

The binary frequency of solar-metallicity dwarfs seem to decrease with the primary mass. Results from visual, speckle, and spectroscopic studies of massive $\mathrm{O}$ and $\mathrm{B}$ stars (isolated or in clusters) have revealed that a significant number are in multiple

\footnotetext{
^ Based on observations collected at the Centro Astronómico Hispano Alemán (CAHA) at Calar Alto, operated jointly by the Max-Planck Institut für Astronomie and the Instituto de Astrofísica de Andalucía (CSIC).
}

systems (59\%, Mason et al. 1998). At lower masses, Duquennoy \& Mayor (1991) have inferred a multiplicity of $57 \pm 9 \%$ for a sample of 164 solar-metallicity stars. These systems have mass ratios larger than 0.1 and harbour a wide range of separations. M dwarf multiples are as frequent as $42 \pm 9 \%$ and have binary properties such as mass ratio and separation similar to G dwarfs (Henry \& McCarthy 1990; Fischer \& Marcy 1992). In the substellar regime (total mass less than $0.1 M_{\odot}$ ), the multiplicity seems lower (10-20\%) with a preference for small separations ( 4-8 AU) and equal-mass systems (Martín et al. 2003; Burgasser et al. 2007).

Population II stars are old (10 Gyr or more), implying that any object more massive than a solar-metallicity star has evolved off the main-sequence into a white dwarf. Independent studies of subdwarfs drawn from the Carney-Latham catalogue (Carney et al. 1994) across a large separation range favour similar binary fractions between metal-poor and solar-metallicity stars with $\mathrm{G}$ spectral types. This conclusion is valid for spectroscopic binaries (<3 AU, Stryker et al. 1985; Latham et al. 2002), separations from a few to tenths of astronomical units (AU, Köhler et al. 2000; Zinnecker et al. 2004), and wide binaries (Allen et al. 2000; Zapatero Osorio \& Martín 2004). In the M dwarf regime, the sample imaged at high resolution is much smaller. Riaz et al. (2008) expanded the Hubble Space Telescope (HST) high-resolution imaging by Gizis \& Reid (2000) and derived an upper limit on the binary frequency of $7 \%(3.6 \pm 3.6 \%$; $1 \sigma$ limit $)$ from a sample of $28 \mathrm{M}$ subdwarfs. An independant sample of 
18 sdM were observed with the Lick adaptive optics Laser Guide Star system by Lépine et al. (2007b) and one sdK7.5, LHS 1589, was resolved into a close binary system, yielding a binary fraction of $5.6 \pm 5.6 \%$.

The fraction of stars hosting planets is larger with higher metallicity. The average metallicity of a volume-limited sample of stars with planets and non binary stars (that have been specifically searched for planets) peaks at about $[\mathrm{Fe} / \mathrm{H}]=+0.1$ and -0.1, respectively (Santos et al. 2005; Bond et al. 2006). The frequency of metal-poor stars with planets is of the order of five percent or less, increasing to $30 \%$ for stars with metallicity of +0.25 (Santos et al. 2001, 2005). This trend is now widely accepted and is not the result of observational effects (e.g., lack of metal-poor stars in the solar neighbourhood or weakness of the absorption lines at lower metallicity). Moreover, there might be a trend towards low-mass planets with short periods orbiting low metallicity stars (Santos et al. 2003; Pinotti et al. 2005).

What is the role of metallicity in the binary properties of low-mass stars? Our aim is to provide a first response to this question and bridge the gap between the high-resolution imaging surveys of metal-poor $G$ stars and radial velocity studies of extrasolar planets around solar-metallicity stars. In this paper we present high-resolution $z$-band imaging for a sample of $24 \mathrm{sdM}$ and nine esdM obtained with the AstraLux camera on the Calar Alto 2.2-m telescope. In Sect. 2 we describe the selection of the sample. In Sect. 3 we present additional photometric and astrometric measurements for the possible faint companion to LHS 1074 detected by Riaz et al. (2008) as well as the observations, data reduction, analysis of the AstraLux data. In Sect. 4 we discuss the binary frequency of early-M subdwarfs and compare our results to previous surveys dedicated to the multiplicity of subdwarfs.

\section{Sample selection}

We have selected the brightest known subdwarfs with spectral types derived from optical spectroscopy by various authors (Table 1). There are 24 subdwarfs and nine extreme subdwarfs with metallicites $[\mathrm{m} / \mathrm{H}]$ of approximately -1.2 and -2.0 , respectively (Gizis 1997). In total, our sample contains 33 metal-poor early-M with spectral types between M0 and M5. They are typically brighter than $I \sim 14 \mathrm{mag}$. Because they are bright, they are usually among the closest $\mathrm{M}$ subdwarfs with distances less than $100 \mathrm{pc}$ (Table 1). However, only four subdwarfs, identified in the SUPERBLINK catalogue (Lépine et al. 2002), are beyond $50 \mathrm{pc}$. Theoretical models predict masses around $\sim 0.1-0.4 M_{\odot}$ and $\sim 0.09-0.2 M_{\odot}$ for early-M subdwarfs and extreme subdwarfs at an age of 10 Gyr (Baraffe et al. 1997), respectively, compared to $\sim 0.13-0.6 M_{\odot}$ for solar-metallicity dwarfs of similar effective temperatures (Baraffe et al. 1998).

Our target list contains seven sources common to a similar survey conducted by Riaz et al. (2008) with the HST, including LHS 491, LHS 320, LHS 3409, LHS 364, LHS 536, LHS 161, and LHS 491 (Luyten 1979). In addition, we have included the LHS 1589AB system (Lépine et al. 2007b) to test the performance of AstraLux and check the common proper motion.

Moreover, we obtained a second imaging epoch with the Long-slit Intermediate Resolution Infrared Spectrograph (LIRIS, Manchado et al. 1998) on the William Herschel Telescope (WHT) of the sdM6 LHS 1074 to investigate the common proper motion of the faint source located two arcsec away from LHS 1074 (Riaz et al. 2008). Both the primary and the candidate are beyond the capabilities of AstraLux. The astrometric study of LHS 1074 is mandatory for a proper statistical analysis of the binary fraction among the low-mass metal-poor stars in the combined surveys by Riaz et al. (2008) and ours. Section 3.1 is dedicated to this object.

\section{Photometric observations}

\subsection{WHT/LIRIS observations of LHS 1074}

In this section we discuss the binarity of LHS 1074, a sdM6.0 subdwarf with a photometric distance estimated as $\sim 86$ pc (Reid \& Gizis 2005) and originally listed in the Luyten Half-Second Catalog (Luyten 1979).

Riaz et al. (2008) imaged LHS 1074 deeply with HST and found a faint companion at $\sim 2.1$ arcsec from the primary. To confirm (or otherwise) the common proper motion of LHS 1074 and the faint companion, we have obtained a deep $J$-band image with LIRIS (Manchado et al. 1998) on the WHT at the Roque de Los Muchachos Observatory on the island of La Palma in the Canaries. LIRIS is equipped with a $1024 \times 1024$ HAWAII detector working in the 0.8 to 2.5 micron wavelength range and has a scale of 0.25 arcsec per pixel, translating into a field of view of 4.27 arcmin a side. LIRIS $J$-band observations were conducted on July 1,2008 . The total integration time was $4860 \mathrm{~s}$. The final stacked image of LHS 1074 consisted of 81 short individual exposures of $60 \mathrm{~s}$. Raw near-infrared data were reduced in a standard way for these wavelengths, including sky substraction and flat-fielding.

The proper motion of LHS 1074 is about $0.8 \mathrm{arcsec} / \mathrm{yr}$ (Luyten 1979). The epoch difference between the HST taken on 24 October 2003 and WHT/LIRIS observations is approximately 4.7 years, long enough to determine unambiguously the common proper motion of LHS 1074 and the possible companion announced by Riaz et al. (2008). On the LIRIS stacked image, we measured a separation of 5.45 arcsec with an uncertainty of one pixel i.e., 0.25 arcsec and a $J$-band magnitude of $21.47 \pm 0.15$ (Table 2). Both results confirm that both objects do not form a physical pair. On the one hand, we expect LHS 1074 to have moved by 3.76 arcsec in 4.7 years which is consistent within the uncertainties with the difference in the separation measured on the HST and LIRIS images. On the other hand, the expected temperature of a subdwarf six magnitudes fainter than its primary, a sdM6 subdwarf, should be very red and substellar. Those objects exhibit redder $z-J$ optical-to-infrared colours (typically 3-4 mag, Hawley et al. 2002; Knapp et al. 2004), inconsistent with the photometry obtained in the $m_{775}(22.6 \mathrm{mag})$ and $J$ filters.

Table 2 lists the coordinates, photometry from $\operatorname{HST}\left(I_{m_{775}}\right)$ for LHS 1074 (top line) and its possible companion (lower line). The $J$-band magnitudes are from 2MASS for LHS 1074 and LIRIS for the companion with an estimated uncertainty of $0.3 \mathrm{mag}$. The respective epochs of the $H S T$ and LIRIS observations are also given (Col. 5) with the measured separations given in arcsec in the last column. The difference in separation matches the proper motion of LHS $1074(\sim 0.8 \mathrm{arcsec} / \mathrm{yr})$ within the measurement uncertainties.

\subsection{AstraLux observations}

Observations were carried out with AstraLux, the "Lucky Imaging" facility installed on the Calar Alto 2.2-m telescope. AstraLux is equipped with a electron-multiplying, thinned, and back-illuminated $512 \times 512 \mathrm{CCD}$ detector with a pixel scale of 47 milli-arcsec (mas), yielding a field-of-view of approximately 24 by 24 arcsec (Hormuth et al. 2008). The read-out 
Table 1. Subdwarfs and extreme subdwarfs observed at high resolution in the $z$-band with AstraLux.

\begin{tabular}{|c|c|c|c|c|c|c|c|c|c|c|c|c|c|c|}
\hline Name & $\begin{array}{c}\text { RA (J2000) } \\
\text { h:m:s }\end{array}$ & $\begin{array}{c}\text { Dec (J2000) } \\
\text { d: : :" } \\
\end{array}$ & $\begin{array}{c}I \\
\text { mag }\end{array}$ & $\begin{array}{c}J \\
\text { mag }\end{array}$ & $\begin{array}{l}\mathrm{PM} \\
1 " / \mathrm{yr}\end{array}$ & $\begin{array}{c}\text { SpT } \\
\ldots \\
\end{array}$ & $\begin{array}{c}d \\
\mathrm{pc}\end{array}$ & Date & $\begin{array}{c}T \\
\mathrm{~ms}\end{array}$ & $\begin{array}{c}\# \text { Exp } \\
\ldots \\
\end{array}$ & Strehl & $\begin{array}{l}\text { Res } \\
\text { pix }\end{array}$ & $\begin{array}{l}20 \mathrm{p} \\
\mathrm{mag}\end{array}$ & $\begin{array}{l}42 p \\
\text { mag }\end{array}$ \\
\hline LSR J0157 & $01: 57: 40.63$ & $+53: 08: 13.2$ & 13.3 & 12.01 & 0.64 & sdM3.5 & 45.0 & 08 Nov. & 50 & 6000 & 14.1 & 2.88 & 3.6 & 4.2 \\
\hline LHS 12 & 02:02:52.16 & $+05: 42: 21.0$ & 11.2 & 9.47 & 2.44 & sdM0.0 & 27.9 & 08 Nov. & 15 & 10000 & 9.0 & 3.20 & 3.0 & 4.1 \\
\hline LHS 156 & 02:34:12.46 & $+17: 45: 50.5$ & 12.9 & 11.43 & 1.18 & sdM3.0 & 36.1 & 08 Nov. & 30 & 10000 & 12.9 & 2.83 & 4.5 & 5.1 \\
\hline LHS 1481 & 02:58:10.23 & $-12: 53: 05.8$ & 99.9 & 8.95 & 0.62 & sdM3.0 & 10.5 & 08 Nov. & 15 & 10000 & 8.9 & 4.00 & 3.4 & 4.6 \\
\hline LHS 174 & $03: 30: 44.82$ & $+34: 01: 07.2$ & 11.1 & 9.84 & 1.57 & $\mathrm{sdM} 0.5$ & 49.0 & 07 Nov. & 30 & 10000 & 13.1 & 4.32 & 3.8 & 5.4 \\
\hline LHS 20 & 03:38:15.58 & $-11: 29: 10.3$ & 99.9 & 9.63 & 0.88 & $\operatorname{sdM} 2.5$ & 15.4 & 08 Nov. & 50 & 6000 & 10.0 & 3.44 & 3.0 & 4.1 \\
\hline LHS 178 & $03: 42: 29.45$ & $+12: 31: 33.8$ & 99.9 & 9.11 & 0.53 & sdM1.5 & 22.2 & 07 Nov. & 15 & 20000 & 14.8 & 4.46 & 4.4 & 6.0 \\
\hline LSR J0342 & 03:42:53.73 & $+55: 27: 30.4$ & 13.8 & 12.88 & 0.50 & sdM0.0 & 150.0 & 08 Nov. & 50 & 6000 & 15.6 & 2.48 & 3.4 & 4.6 \\
\hline LHS 211 & 05:48:00.19 & $+08: 22: 14.2$ & 12.3 & 11.19 & 1.27 & sdM0.0 & 53.2 & 16 Jan., 09 Nov. & 30 & 10000 & 16.2 & 3.27 & 4.1 & 5.6 \\
\hline LSR J0618 & $06: 18: 52.54$ & $+16: 14: 56.0$ & 12.9 & 12.74 & 0.65 & $\operatorname{sdM} 2.0$ & 85.0 & 16 Jan., 07 Nov. & 50 & 6000 & 14.0 & 2.32 & 3.8 & 4.5 \\
\hline LSR J0705 & 07:05:48.77 & $+05: 06: 17.3$ & 13.4 & 13.69 & 0.51 & sdM3.5 & 90.0 & 07 Nov. & 50 & 10000 & 18.1 & 2.01 & 4.6 & 4.7 \\
\hline LHS 307 & $11: 32: 45.28$ & $+43: 59: 44.5$ & 13.3 & 12.25 & 1.15 & $\mathrm{sdM} 0.5$ & 54.6 & 16 Jan., 09 Nov. & 50 & 6000 & 12.0 & 2.41 & 3.9 & 4.2 \\
\hline LHS 42 & $11: 40: 20.26$ & $+67: 15: 35.0$ & 11.4 & 09.41 & 3.17 & sdM0.0 & 30.6 & 09 Nov. & 30 & 10000 & 10.7 & 3.46 & 3.7 & 4.8 \\
\hline LHS 2497 & 12:02:18.19 & $+28: 35: 14.3$ & 10.2 & 09.13 & 0.79 & sdM3.5 & 20.3 & 09 Nov. & 15 & 10000 & 11.2 & 4.43 & 3.9 & 5.4 \\
\hline LHS 320 & 12:02:33.65 & $+08: 25: 50.6$ & 11.6 & 10.74 & 1.18 & $\operatorname{sdM} 2.0$ & 38.5 & 09 Nov. & 30 & 10000 & 11.4 & 3.33 & 3.4 & 4.9 \\
\hline LSR J1755 & $17: 55: 32.76$ & $+16: 48: 59.0$ & 12.5 & 11.35 & 0.99 & sdM3.5 & 28.0 & 04 Jun., 09 Nov. & 50 & 6000 & 8.8 & 2.28 & 3.9 & 4.2 \\
\hline LHS 3409 & $18: 45: 52.37$ & $+52: 27: 40.0$ & 12.9 & 10.97 & 0.85 & sdM4.5 & 20.0 & 04 Jun., 09 Nov. & 50 & 6000 & 10.7 & 3.23 & 3.9 & 4.8 \\
\hline LSR J1914A & 19:14:05.50 & $+28: 25: 52.3$ & 13.6 & 13.53 & 0.53 & sdM0.0 & 200.0 & 07 Nov. & 50 & 5000 & 16.9 & 2.39 & 4.2 & 4.6 \\
\hline LHS 479 & $19: 46: 48.60$ & $+12: 04: 58.1$ & 12.9 & 11.20 & 1.48 & sdM1.0 & 44.6 & 04 Jun., 09 Nov. & 50 & 6000 & 12.3 & 3.63 & 3.0 & 4.1 \\
\hline LHS 482 & 20:05:02.28 & $+54: 26: 03.8$ & 12.3 & 08.83 & 1.47 & sdM1.5 & 53.2 & 04 Jun., 09 Nov. & 50 & 10000 & 14.2 & 2.93 & 3.7 & 5.1 \\
\hline LSR J2009 & 20:09:33.82 & $+56: 59: 25.8$ & 13.1 & 11.86 & 0.82 & $\operatorname{sdM} 2.0$ & 55.0 & 05 Jun., 09 Nov. & 50 & 6000 & 15.6 & 3.03 & 4.1 & 4.9 \\
\hline LHS 491 & 20:27:29.06 & $+35: 59: 24.5$ & 12.4 & 11.60 & 1.31 & sdM1.5 & 47.4 & 04 Jun., 09 Nov. & 50 & 6000 & 14.5 & 3.27 & 3.9 & 5.0 \\
\hline LHS 64 & 21:07:55.43 & $+59: 43: 19.9$ & 10.9 & 10.12 & 2.11 & sdM1.5 & 23.9 & 05 Jun., 09 Nov. & 15 & 10000 & 14.9 & 3.53 & 4.5 & 5.7 \\
\hline LHS 536 & 23:08:26.08 & $+31: 40: 24.0$ & 12.1 & 11.71 & 1.52 & sdM0.5 & 44.0 & 09 Nov. & 50 & 6000 & 13.6 & 3.14 & 4.0 & 5.0 \\
\hline LHS 161 & $02: 52: 45.51$ & $+01: 55: 50.6$ & 12.7 & 11.71 & 1.55 & esdM2.0 & 38.5 & 07 Nov. & 30 & 10000 & 14.5 & 2.79 & 4.3 & 5.2 \\
\hline LHS 182 & 03:50:13.89 & $+43: 25: 40.5$ & 12.6 & 11.10 & 1.44 & esdM0.0 & 42.7 & 07 Nov. & 50 & $6000^{a}$ & 9.7 & 2.48 & 3.4 & 3.6 \\
\hline LHS 185 & 04:01:36.60 & $+18: 43: 39.9$ & 13.0 & 14.63 & 1.17 & esdM0.5 & 59.9 & 07 Nov. & 50 & 6000 & 13.4 & 2.63 & 4.0 & 4.8 \\
\hline LHS 1742 & 05:10:31.41 & $+31: 17: 35.4$ & 14.3 & 99.99 & 0.83 & esdM5.5 & 11.2 & 07 Nov. & 50 & 6000 & 10.5 & 3.01 & 3.6 & 4.5 \\
\hline LSR J0627 & $06: 27: 33.31$ & $+06: 16: 58.8$ & 99.9 & 13.29 & 1.02 & esdM1.5 & 80.0 & 16 Jan., 07 Nov. & 50 & 6000 & 16.9 & 2.15 & 4.2 & 4.7 \\
\hline LHS 364 & 14:06:55.54 & $+38: 36: 57.8$ & 12.9 & 11.47 & 1.05 & esdM1.5 & 26.7 & 16 Jan. & 50 & 6000 & 10.7 & 4.02 & 4.6 & 4.8 \\
\hline LHS 3382 & $18: 21: 52.95$ & $+77: 09: 30.1$ & 14.0 & 13.86 & 0.77 & esdM2.5 & 96.2 & 09 Nov. & 50 & 6000 & 18.8 & 1.94 & 5.1 & 5.3 \\
\hline LHS 489 & 20:19:04.58 & $+12: 35: 04.1$ & 13.9 & 12.53 & 1.24 & esdM0.0 & 52.9 & 09 Nov. & 50 & 6000 & 14.6 & 2.58 & 4.1 & 4.8 \\
\hline LSR J2205 & $22: 05: 32.78$ & $+58: 07: 26.8$ & 99.9 & 12.12 & 0.54 & esdM1.0 & 70.0 & 05 Jun., 09 Nov. & 50 & $6000^{b}$ & 18.1 & 2.78 & 4.1 & 4.9 \\
\hline
\end{tabular}

Table 2. Coordinates, photometry, epochs of observations, and separations from the HST (1) and LIRIS observations (2).

\begin{tabular}{cccccc}
\hline \hline $\begin{array}{c}\text { RA (J2000) } \\
\text { h:m:s }\end{array}$ & $\begin{array}{c}\text { Dec (J2000) } \\
\text { d: : : }\end{array}$ & $\begin{array}{c}I_{m_{775}} \\
\text { mag }\end{array}$ & $\begin{array}{c}J_{\mathrm{C}} \\
\text { mag }\end{array}$ & $\begin{array}{c}\text { Epoch } \\
\text { years }\end{array}$ & $\begin{array}{c}\text { Sep } \\
\operatorname{arcsec}\end{array}$ \\
\hline $00: 25: 51.32$ & $-07: 48: 09.3$ & 16.07 & 14.68 & 2003.81 & 2.1 \\
00:25:51.31 & $-07: 48: 11.4$ & 22.60 & 21.47 & 2008.50 & 5.45 \\
\hline
\end{tabular}

References: (1) Riaz et al. (2008); (2) this paper.

noise is of the order of 80 electrons and the distortion across the entire field is less than $0.5 \%$. Various optical filters are available for observations, including the Cousins $I$ (Johnson \& Morgan 1953; Cousins 1978) and the SDSS (Sloan Digital Sky Survey) $i$ and $z$ (Fukugita et al. 1996).

We have targeted a total of $24 \mathrm{sdM}$ and nine esdM over three campaigns (Table 1): 14 and 16 January, 04-05 June, and 07-10 November 2008. In January, the nights were clear with seeing around 1.5 arcsec. The night of June 04th was affected by clouds despite a seeing in the $0.8-1.2$ arcsec range. The following night was clear with good transparency but the seeing oscillated significantly with values up to 2 arcsec. In November, the conditions were significantly better than in January and June with clear skies and seeing around $0.6-0.8$ arcsec. Therefore, we decided to repeat all the subdwarfs observable in November to improve the depth of the images. Typically, we obtained a total of $300 \mathrm{~s}$ total integration time divided into 6000 exposures of 50 milli-seconds ( $\mathrm{ms}$ ) for our targets to sample the rapid seeing variations. However, those values were modified accordingly depending on the brightness of the source. The Strehl ratios are usually higher than $10 \%$, except for a few targets (Table 1). We have selected the $10 \%$ best-quality images resulting in a effective $30 \mathrm{~s}$ exposure time. We have achieved resolutions of $\sim 0.1 \mathrm{arcsec}$, typical for Lucky imaging observations in a 2-m class telescope, for all the subdwarfs (Table 1), i.e., about one-tenth of the natural seeing conditions at the time of the observations.

A series of dome flat fields and bias were taken before each night and skyflats after sunset when possible to create master bias and master flats. We have also observed the M 15 globular cluster during each night to calculate the pixel scale and the orientation of the camera and derive the photometric zero-points (applied only if a binary was found).

\subsection{Data reduction}

The data reduction of the raw images are done on-the-fly with an automatic pipeline available at the telescope and distributed by the AstraLux team (Hormuth et al. 2008). In the case of the sole binary resolved by our survey, the comparable brightness measured for the primary and the secondary leads to a "triple" system with the automatic pipeline. Therefore, we re-processed the raw images by imposing the primary star as the reference to remove this well-known effect present in Lucky Imaging images. This was done using the "two star" option available in the FastCam (PI Rafael Rebolo) ${ }^{1}$ data reduction package developed

\footnotetext{
1 More details on FastCam at: http://www.iac.es/proyecto/ fastcam/
} 


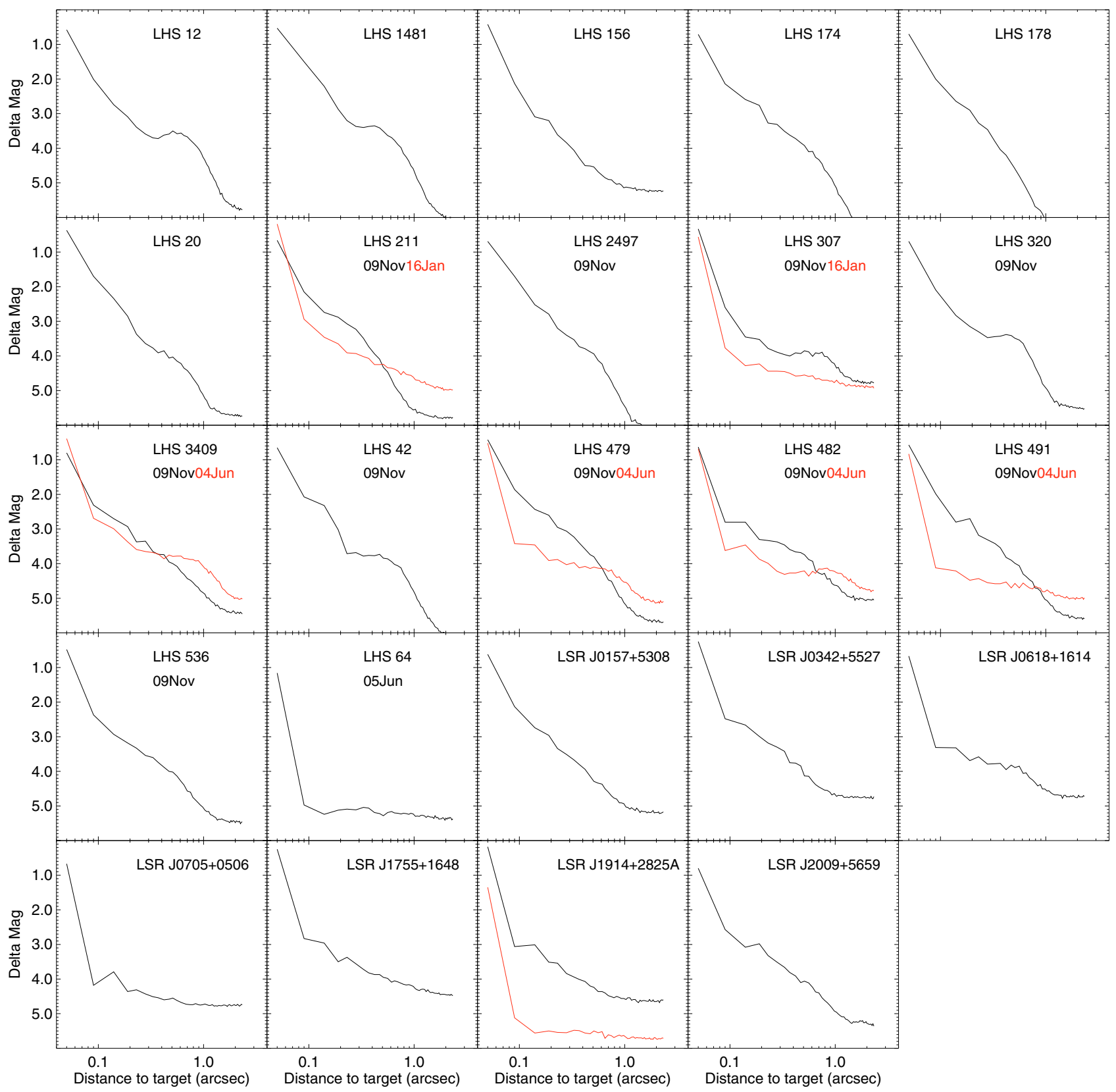

Fig. 1. Difference in magnitude (Delta Mag) between the subdwarf targets and the sky as a function of separation from the primary for the best $10 \%$ of all AstraLux images.

by the Universidad Polytécnica de Cartagena. A similar result was obtained by Felix Hormuth (personal comm.) using a simple "detripling" algorithm implemented to remove a "triple" component when the primary and the secondary of a binary system have comparable brightness. Although we have not been involved in the writing of the automatic pipeline, we give a short summary of the steps involved in the data processing. More specific information on the processing is extensively detailed in Hormuth et al. (2008) and in the PhD theses of Robert Tubbs and Nicholas Law $^{2}$ as well as on the AstraLux website ${ }^{3}$.

All the observations were taken using only one-fourth of the detector, covering a 6 by 6 arcsec field-of-view. Firstly, the raw $z$-band images were divided by the master flats previously

\footnotetext{
2 http://wWw.ast.cam.ac.uk/ optics/Lucky_Web_Site/ references.htm

${ }^{3}$ http://www . mpia.de/ASTRALUX/Publications.html
}

corrected for the bias. Secondly, the quality of the images was determined by measuring the frames with the largest flux in the point-spread function of the reference star. In our case, the target was used as the reference star and was usually (but not always) the only object in the field. The next step consists of computing the shifts between each individual frame to align all the images. Then, the frames are ranked on the basis of the quality of their point-spread function. The $1,2.5,5$, and $10 \%$ bestquality images were aligned, and co-added using the Drizzle algorithm (Fruchter \& Hook 2002) to provide a final pixel scale of 0.0233 mas. Finally, those images are saved to disk for visual inspection. The pipeline processing is quicker than the typical exposure time used for our targets and allowed us to obtain an $i$-band image of any potential binary system before moving to the next target. 


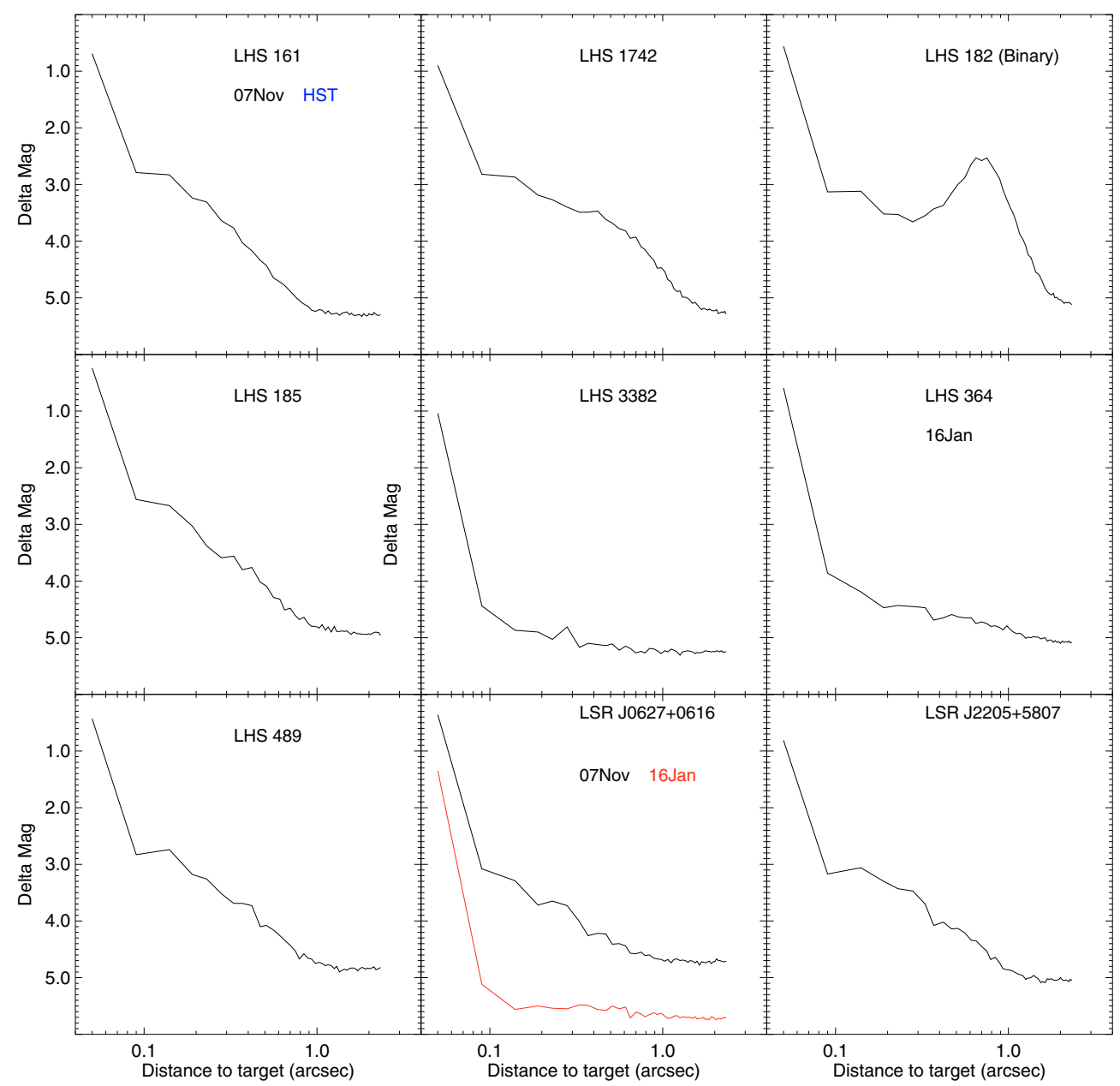

Fig. 2. Difference in magnitude (Delta Mag) between the extreme subdwarf targets and the sky as a function of separation from the primary for the best $10 \%$ of all AstraLux images.

\subsection{Analysis}

The selection of the best $10 \%$ of the images provides diffraction-limited images for 2-m class telescopes i.e., fullwidth-half-maximum (FWHM) of the order of 0.1 arcsec at red (0.6-0.9 microns) optical wavelengths. We have measured the number of counts from the sky at different radii from the object to estimate the depth achieved on each image as a function of the distance from the target $(\Delta \mathrm{Mag})$. We incremented the radii by 2 pixels and assumed a pixel scale of 0.0233 pixel resulting from the Drizzled algorithm applied to the AstraLux images. We typically achieved a $\Delta \mathrm{Mag}$ of $\sim 2 \mathrm{mag}$ at $\sim 0.1$ arcsec from the target and 4-5 mag at $\sim 1.0$ arcsec (Table 1). We are sensitive to separations as large as $300 \mathrm{AU}$ at a distance of $50 \mathrm{pc}$, the size of the AstraLux detector (6 arcsec in each direction). The results are shown in Figs. 1 and 2 for the $24 \mathrm{sdM}$ and 9 esdM, respectively. Figure 2 includes the new binary system, LHS 182, resolved with AstraLux. We give the $\Delta \mathrm{Mag}$ at 0.47 and 0.98 arcsec (corresponding roughly to 20 and 40 pixels, respectively) from the target in Table 1 . Note that several objects were observed on two different nights and we list the best $\Delta$ Mag for them in Table 1 but we show the depth achieved as a function of the separation to the primary for both nights (Figs. 1, 2).

To assess the depth and resolution achieved with the AstraLux images, we have compared our sensitivity curves with HST observations of seven subdwarfs observed by Riaz et al. (2008) and common to our sample. The depth within 0.5 arcsec of the target is greater in HST observations than in our groundbased images (typically 6 vs. $2 \mathrm{mag}$ ). The depth by the $H S T$ at larger distances is typically three magnitudes better than for our AstraLux data. The range of resolution and depth achieved by the Lucky Imaging technique lie within the sensitivity figures of the HST, implying that we can add the sample of subdwarfs targeted by Riaz et al. (2008) to our sample to discuss the binary properties of low-metallicity M dwarfs.

We have found objects close to our targets on the AstraLux images in three cases but the companionship is unlikely in two cases, LHS 489 and LSR J2009+5659, due to the large proper motion of the objects (Fig. 3). In the former case, LHS 489 is moving southwards $\left(\mu_{\alpha} \cos \delta=-0.053, \mu_{\delta}=-1.219\right.$ arcsec/yr) towards two stars oriented north-south and separated by about four arcsec. The expected position of LHS 489 at the epoch of the AstraLux observations based on its proper motion is consistent with a separation of $\sim 2$ arcsec with star \#1 (top panel in Fig. 3). We have repeated a similar analysis using the proper motion of LSR J2009+5659 $\left(\mu_{\alpha} \cos \delta=+0.431\right.$; $\left.\mu_{\delta}=+0.700 \operatorname{arcsec} / \mathrm{yr}\right)$ and determined that it should now be located north-east of 2MASS J200934.16+565930.2 (source \# 1 in bottom panels of Fig. 3) during our November observing run, consistent with the position on the AstraLux images.

Figure 3 displays the image showing the motions of LHS 489 (top) and LSR J2009+5659 over 55 years on POSS I (year 1953; left), POSS II (year 1990; middle), and AstraLux images (SDSSz; November 2008; right). The size of the POSS images 


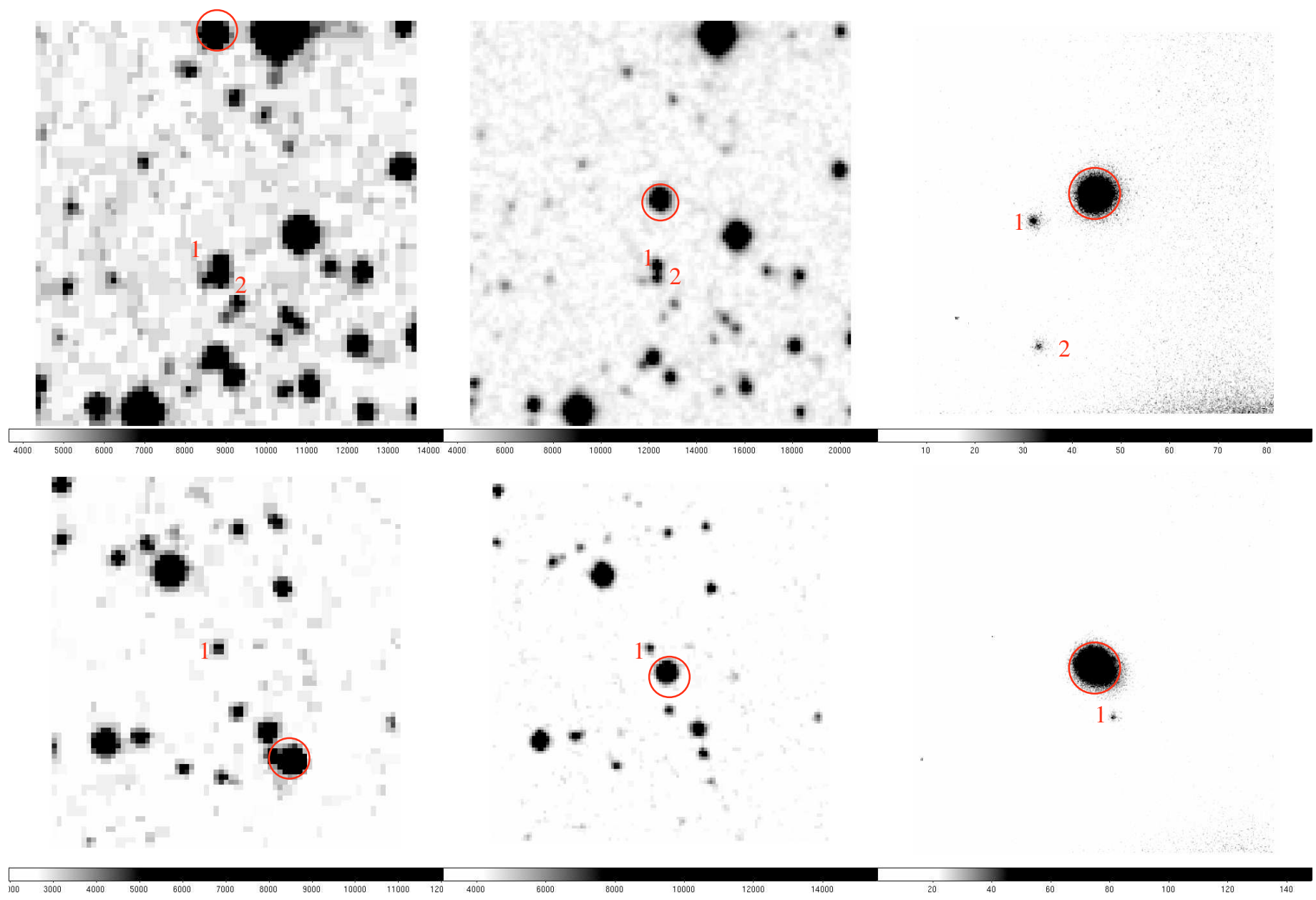

Fig. 3. Images showing the motion of LHS 489 (top) and LSR J2009+5659 over 55 years on POSS I (year 1953; left), POSS II (year 1990; middle), and AstraLux images (SDSSz; November 2008; right).

Fig. 4. AstraLux images of the LHS 1589AB system in the SDSS $i$ (left) and $z$ (right) filters.

is two arcmin across whereas the AstraLux images are 12 arcsec wide. The objects marked with numbers and detected on the AstraLux images are unlikely to be physically associated with the targets (circled) because they were already present on the photographic plates and appear closer to the target due to the large motions of LHS 489 (1.22 arcsec/yr) and LSR J2009+5659 (0.822 $\mathrm{arcsec} / \mathrm{yr})$.

We verify the astrometric companionship of LHS 1589A\&B discovered with the Lick adaptive optics laser guide star system by Lépine et al. (2007b). The epoch difference between

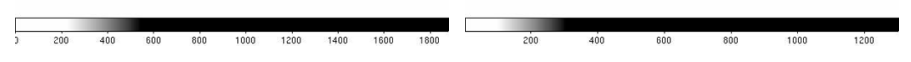

Fig. 5. AstraLux images of LHS 182 in the SDSS $i$ (left) and $z$ (right) filters.

the Lick (16/17 September 2006) and the latest AstraLux (November 2008) observations is greater than two years. We have measured a separation of 11 pixels or $0.25 \pm 0.02$ arcsec (Fig. 4). Figure 4 displays the AstraLux images of the LHS 1589AB system (Lépine et al. 2007b) in the SDSS $i$ (left) and $z$ (right) filters. The pixel scale is 23.3 mas and the field-ofview is about 3.5 arcsec aside with North up and East left. The separation of $0.224 \pm 0.004$ arcsec measured by Lépine et al. (2007b) is in agreement with the separation on the AstraLux images $(0.25 \pm 0.02)$, confirming the common proper motion of the system. 
During this period, LHS 1589A has moved by more than 1.6 arcsec, implying that the separation should have increased significantly if the companion was not associated with LHS $1589 \mathrm{~A}^{4}$. As a consequence, we confirm the LHS $1589 \mathrm{AB}$ pair as a true metal-poor $([m / H] \sim-1.0)$ and low-mass $\left(0.3 M_{\odot}\right)$ binary system (Lépine et al. 2007b).

\subsection{LHS 182: an extreme subdwarf binary}

Observations in November 2008 of LHS 182 (esdM0; $d=$ 42.7 pc, Harrington \& Dahn 1980) revealed a close companion at $\sim 0.7$ arcsec (Fig. 5), an object detected neither on photographic plates nor in 2MASS (Cutri et al. 2003) after taking into account the proper motion of LHS $182\left(\mu_{\alpha}=-0.374\right.$, $\mu_{\delta}=-1.391 \mathrm{arcsec} / \mathrm{yr}$, Lépine \& Shara 2005) as we did for e.g. LHS 489. Figure 5 shows the SDSS $i$ (left) and $z$ (right) filters mounted on AstraLux on the Calar Alto 2.2-m telescope. The pixel scale is 23.3 mas and field-of-view is about three arcsec aside with North up and East left.

The closest objects detected in previous surveys are located at a few arcsec from the position of LHS 182 in November 2008. Therefore, we consider this system as a true physical binary. After re-processing the raw images to remove the effect of the "triple" system (see details in Sect. 3.3), we have measured a brightness ratio of $0.1-0.2 \mathrm{mag}$ in the $z$-band and a separation of $0.70 \pm 0.05$ arcsec, corresponding to a projected physical separation of about $30 \mathrm{AU}$ at the distance of LHS 182. An image taken three months later (on 13 February 2009) with AstraLux confirms the common proper motion of the pair and the separation of $\sim 0.7$ arcsec. We also note that this pair was reported by Jao et al. (2009) and a similar separation was reported (0.62 arcsec) from observations made in November 2005.

\section{Binary fraction of early-M subdwarfs}

In this section we place our results in a wider context and discuss the possible role played by the metallicity in the binary properties of low-mass stars.

We have detected one close companion to LHS 182, an esdM0 at $\sim 43 \mathrm{pc}$, from a sample of 33 metal-poor early-M dwarfs (Table 1). We have shown in Sect. 3.4 that we are able to resolve LHS 1589AB into a binary system with a separation of $\sim 0.2$ arcsec and a difference in $K$ of $0.52 \mathrm{mag}$ (Lépine et al. 2007b), suggesting that the resolution obtained with AstraLux is comparable to high-resolution imaging surveys with adaptive optics and from space. From our AstraLux sample alone, we infer a binary fraction of $1 / 33=3 \pm 3 \%$ (Poissonian errors) at separations wider than $5 \mathrm{AU}$ around metal-poor early-M dwarfs (assuming a mean distance of $50 \mathrm{pc}$ ). This binary frequency is comparable to the fraction derived by Riaz et al. (2008) from a sample of 28 objects $(3.6 \pm 3.6 \%$; we have shown in Sect. 3.1 that the companion found around LHS 1074 does not share the same proper motion) and by Lépine et al. (2007b) from 18 subdwarfs $(5.6 \pm 5.6 \%)$. We note that the only binary found by Riaz et al. (2008) is not a subdwarf binary but a wide common proper motion pair composed of a subdwarf and a white dwarf (LHS 2139/2140, Luyten 1979). Adding the 28 subdwarfs from Riaz et al. (2008) to our sample (and taking into account the seven objects in common), the fraction of $0.1-0.4 M_{\odot}$ subdwarf binaries is $2 /(33+28-7)=3.7 \pm 2.6 \%(1 \sigma$ confidence level $)$ for the aforementioned separation range. The binary fraction

\footnotetext{
${ }_{4}$ We also observed LHS 1589AB in November 2007 and measured a separation of $\sim 0.2$ arcsec.
}

of solar-metallicity $\mathrm{M}$ dwarfs with masses in the $0.13-0.6 M_{\odot}$ range is 20-25\% (Fischer \& Marcy 1992; Reid \& Gizis 1997) for separations in the 6-300 AU range. Therefore, there is a significant difference (a factor of two with a $3 \sigma$ confidence level) between the binary frequency of metal-poor early-M dwarfs and their solar counterparts in that separation range. Below we discuss the possible reasons for this discrepancy and the role that metallicity seems to play at lower masses. There are several alternatives to account for the deficit of binary systems at low metallicity.

One possible explanation could reside in the difference in the composition of the metal-poor and solar-metallicity parent clouds, leading to distinct binary properties. However, metalpoor and solar-metallicity stars seem to share the binary characteristics (frequency and orbital parameters) over the entire separation range for masses above $\sim 1 M_{\odot}$ (Partridge 1967; Stryker et al. 1985; Allen et al. 2000; Latham et al. 2002; Zapatero Osorio \& Martín 2004). Surveys involving thousands of radial velocity measurements of hundredth of stars (Carney et al. 1994) taken over several years (Stryker et al. 1985; Latham et al. 2002) concluded that the frequency of spectroscopic binaries for both metal-poor and solar-metallicity $\mathrm{G}$ stars is around $15 \%$ (Duquennoy \& Mayor 1991), in agreement with the early study of Partridge (1967). At very wide projected physical separation $(a>25 \mathrm{AU})$, the picture seems comparable with $15 \%$ of subdwarfs exhibiting companions (Allen et al. 2000; Zapatero Osorio \& Martín 2004). At intermediate separations, the frequency of metal-poor stars seems again in agreement with the fraction of solar-metallicity G stars (Zinnecker et al. 2004). As pointed out by Latham et al. (2002), if the composition of the initial molecular cloud plays a role in setting the binary properties, it is unlikely to be the main reason for the deficit of metal-poor lowmass stars.

Nonetheless, metallicity may play a role early on in the formation of such multiple systems as appears to be the case for the formation of massive planets around solar-mass stars. This fact is supported by the higher number of planets orbiting metalrich stars than solar-metallicity stars (Santos et al. 2005; Bond et al. 2006) coupled with the non-detection of planets in 47 Tuc ( $\mathrm{Fe} / \mathrm{H}=-0.7$, Gilliland et al. 2000). The amount of metals in the molecular clouds would favour the presence of solid planetesimals on which dust accumulates to grow and ultimately form giant planets (Santos et al. 2001). However, this process is still not very well understood.

Metal-poor stars tend to be older than solar-abundance stars and have thus suffered more encounters with other stars. The large majority may stem from globular clusters whose high density favour the disruption of binaries with separations larger than a few AU. Therefore, we are measuring the results of dynamical interactions between stars in dense environments. However, the tightest systems with the highest binding energy should be less affected by the process of ejection (Sterzik \& Durisen 1998), pointing in the direction of a higher binary frequency for solarmass stars than for $\mathrm{M}$ dwarfs. If this assumption is true, radial velocity surveys should find a higher frequency for spectroscopic binaries than our results although it may remain lower than the fraction inferred for solar-metallicity $\mathrm{M}$ dwarfs. This effect can be compared with the difference observed between the binary frequency of low-mass stars in the dense Orion cluster and the low-density Taurus star-forming region (Köhler et al. 2006): they observed a factor of 3-5 difference between both clusters. However, they found that the binary fraction is roughly constant when going radially out from the centre, suggesting that disruption is not the main effect. The most likely explanation is 
the extremely dense environment dominating during the formation process of massive open clusters like the Trapezium Cluster (Kroupa et al. 2001). This effect would be magnified for globular clusters and could lead to the difference observed between metal-poor and solar-metallicity M dwarfs (e.g., the lack of planets in 47 Tuc, Gilliland et al. 2000).

Moreover, low-metallicity stars could populate the Milky Way as a result of mergers with satellite galaxies. For example, Meza et al. (2005) and Abadi et al. (2006) presented simulations predicting that a large number of nearby metal-poor stars would originate from a merger with a satellite galaxy like $\omega$ Cen. They would then remain in the Galaxy after suffering significant perturbations that would likely modify their primordial binary properties, which are unknown. Besides, the environment in those galaxies could be very different to what we know in our Galaxy.

Lastly, another potential explanation could be that the massluminosity relation for low-mass stars and brown dwarfs cools down faster than predicted by theoretical models and/or is steeper for a given age. This interpretation, although less probable than the aforementioned proposals, cannot be discarded with current data.

\section{Conclusions}

We have presented high-resolution imaging for a sample of $33 \mathrm{M}$ subdwarfs located within $100 \mathrm{pc}$ of the Sun. We have uncovered one close (projected physical separation of 30 AU) companion to the M0 extreme subdwarf LHS 182. No companion was resolved around the other targets at separations larger than 10 and $50 \mathrm{AU}$ (at a distance of $50 \mathrm{pc}$ ) down to two and five magnitudes fainter than the primary, respectively. Moreover, we do not confirm the common proper motion candidate of LHS 1074 discovered by Riaz et al. (2008) on astrometric and photometric grounds. Our results support previous surveys of $\mathrm{M}$ subdwarfs and suggests that the binary frequency of $\mathrm{M}$ dwarfs is metallicity-dependent.

To test whether or not subdwarfs are born in globular clusters and survive the high densities present at the early formation stage, radial velocity surveys of a large sample of $\mathrm{M}$ subdwarfs and extreme subdwarfs should be conducted. The main drawback is the large amount of time required on 8 to 10-m class telescopes to achieve good signal-to-noise ratios at those faint magnitudes. However, such a survey would shed light on the origin of halo interlopers. Finally, many ultracool subdwarfs have been discovered recently (e.g., Lépine \& Scholz 2008) and this rate will likely increase with current (e.g., UKIDSS) and upcoming large-scale surveys (Visible and Infrared Survey Telescope for Astronomy $^{5}$; PanSTARS ${ }^{6}$; Large Synoptic Survey Telescope ${ }^{7}$ ). Finding metal-poor brown dwarfs and investigating their binary properties will require large telescopes combined with Laser Guide Star facilities.

Acknowledgements. Support for this project has been provided by the Spanish Ministry of Science via project AYA2007-67458. Thanks to the Calar Alto staff for their support with the AstraLux observations and Felix Hormuth for valuable information. N.L. thanks Luisa Valdivielso and Florian Rodler for their assistance with the Lucky Imaging observations. This research has made use of the Simbad database, operated at the Centre de Données Astronomiques de Strasbourg (CDS), and of NASA's Astrophysics Data System Bibliographic Services (ADS). This publication makes use of data products from the Two Micron All Sky Survey (2MASS), which is a joint project of the University of Massachusetts and the Infrared Processing and Analysis Center/California
Institute of Technology, funded by the National Aeronautics and Space Administration and the National Science Foundation.

\section{References}

Abadi, M. G., Navarro, J. F., \& Steinmetz, M. 2006, MNRAS, 365, 747

Allen, C., Poveda, A., \& Herrera, M. A. 2000, A\&A, 356, 529

Baraffe, I., Chabrier, G., Allard, F., \& Hauschildt, P. H. 1997, A\&A, 327, 1054

Baraffe, I., Chabrier, G., Allard, F., \& Hauschildt, P. H. 1998, A\&A, 337, 403

Bond, J. C., Tinney, C. G., Butler, R. P., et al. 2006, MNRAS, 370, 163

Burgasser, A. J., Reid, I. N., Siegler, N., et al. 2007, in Protostars and Planets V, ed. B. Reipurth, D. Jewitt, \& K. Keil, 427

Carney, B. W., Latham, D. W., Laird, J. B., \& Aguilar, L. A. 1994, AJ, 107, 2240

Cousins, A. W. J. 1978, Monthly Notes of the Astronomical Society of South Africa, 37, 8

Cutri, R. M., Skrutskie, M. F., van Dyk, S., et al. 2003, 2MASS All Sky Catalog of point sources, 2246

Duquennoy, A., \& Mayor, M. 1991, A\&A, 248, 485

Fischer, D. A., \& Marcy, G. W. 1992, ApJ, 396, 178

Fruchter, A. S., \& Hook, R. N. 2002, PASP, 114, 144

Fukugita, M., Ichikawa, T., Gunn, J. E., et al. 1996, AJ, 111, 1748

Gilliland, R. L., Brown, T. M., Guhathakurta, P., et al. 2000, ApJ, 545, L47

Gizis, J. E. 1997, AJ, 113, 806

Gizis, J. E., \& Reid, I. N. 2000, PASP, 112, 610

Harrington, R. S., \& Dahn, C. C. 1980, AJ, 85, 454

Hawley, S. L., Covey, K. R., Knapp, G. R., et al. 2002, AJ, 123, 3409

Henry, T. J., \& McCarthy, D. W. 1990, ApJ, 350, 334

Hormuth, F., Hippler, S., Brandner, W., Wagner, K., \& Henning, T. 2008, in Proc. SPIE Conf. 7014

Jao, W.-C., Henry, T. J., Beaulieu, T. D., \& Subasavage, J. P. 2008, AJ, 136, 840 Jao, W.-C., Mason, B. D., Hartkopf, W. I., Henry, T. J., \& Ramos, S. N. 2009, AJ, 137, 3800

Johnson, H. L., \& Morgan, W. W. 1953, ApJ, 117, 313

Knapp, G. R., Leggett, S. K., Fan, X., et al. 2004, AJ, 127, 3553

Köhler, R., Zinnecker, H., \& Jahreiß, H. 2000, in Birth and Evolution of Binary Stars, Poster Proc. IAU Symp. on The Formation of Binary Stars, held 1015 April, 2000, in Potsdam, Germany, ed. B. Reipurth, \& H. Zinnecker, IAU Symp., 200, , 148

Köhler, R., Petr-Gotzens, M. G., McCaughrean, M. J., et al. 2006, A\&A, 458, 461

Kroupa, P., Aarseth, S., \& Hurley, J. 2001, MNRAS, 321, 699

Latham, D. W., Stefanik, R. P., Torres, G., et al. 2002, AJ, 124, 1144

Lépine, S., \& Scholz, R.-D. 2008, ApJ, 681, L33

Lépine, S., \& Shara, M. M. 2005, AJ, 129, 1483

Lépine, S., Shara, M. M., \& Rich, R. M. 2002, AJ, 124, 1190

Lépine, S., Rich, R. M., \& Shara, M. M. 2007a, ApJ, 669, 1235

Lépine, S., Rich, R. M., Shara, M. M., Cruz, K. L., \& Skemer, A. 2007b, ApJ, 668,507

Luyten, W. J. 1979, LHS catalogue. A catalogue of stars with proper motions exceeding $0^{\prime \prime} 5$ annually, Minneapolis, University of Minnesota, 2nd edn.

Manchado, A., Fuentes, F. J., Prada, F., et al. 1998, in Proc. SPIE Conf. 3354, ed. A. M. Fowler, 448

Martín, E. L., Barrado y Navascués, D., Baraffe, I., Bouy, H., \& Dahm, S. 2003, ApJ, 594, 525

Mason, B. D., Gies, D. R., Hartkopf, W. I., et al. 1998, AJ, 115, 821

Meza, A., Navarro, J. F., Abadi, M. G., \& Steinmetz, M. 2005, MNRAS, 359, 93 Partridge, R. B. 1967, AJ, 72, 713

Pinotti, R., Arany-Prado, L., Lyra, W., \& Porto de Mello, G. F. 2005, MNRAS, 364, 29

Reid, I. N., \& Gizis, J. E. 1997, AJ, 113, 2246

Reid, I. N., \& Gizis, J. E. 2005, PASP, 117, 676

Riaz, B., Gizis, J. E., \& Samaddar, D. 2008, ApJ, 672, 1153

Santos, N. C., Israelian, G., \& Mayor, M. 2001, A\&A, 373, 1019

Santos, N. C., Israelian, G., Mayor, M., Rebolo, R., \& Udry, S. 2003, A\&A, 398, 363

Santos, N. C., Israelian, G., Mayor, M., et al. 2005, A\&A, 437, 1127

Sterzik, M. F., \& Durisen, R. H. 1998, A\&A, 339, 95

Stryker, L. L., Hesser, J. E., Hill, G., Garlick, G. S., \& Okeefe, L. M. 1985, PASP, 97, 247

Zapatero Osorio, M. R., \& Martín, E. L. 2004, A\&A, 419, 167

Zinnecker, H., Köhler, R., \& Jahreiß, H. 2004, in Rev. Mex. Astron. Astrofis. Conf. Ser. 21, ed. C. Allen, \& C. Scarfe, 33

\footnotetext{
5 http://www.vista.ac.uk/

6 http://pan-starrs.ifa.hawaii.edu/public/

7 http://www. 1sst.org/
} 